\title{
Synthetic apomixis an old enigma to preserve hybrid vigor
}

Sajid Fiaz ${ }^{1 *}$, Xiukang Wang ${ }^{2 *}$, Rizwana Maqbool ${ }^{3}$, Habib Ali ${ }^{4}$, Shakeel Ahmad ${ }^{5}$, Adeel Riaz ${ }^{6}$, Badr Alharthi ${ }^{7 ’ 8}$

${ }^{1}$ Department of Biochemistry, University of Okara, Punjab, Pakistan

${ }^{2}$ College of Life Sciences, Yan'an University, Yan'an 716000, Shaanxi, China

${ }^{3}$ Department of Plant Breeding and Genetics, Sub Campus Depalpur Okara, University of Agriculture Faisalabad, Punjab, Pakistan

${ }^{4}$ Department of Entomology, Sub Campus Depalpur Okara, University of Agriculture Faisalabad, Punjab, Pakistan

${ }^{5}$ State Key Laboratory of Rice Biology, China National Rice Research Institute, Hangzhou,

Zhejiang, China

${ }^{6}$ Biotechnology Research Institute, Chinese Academy of Agricultural Sciences, Beijing 100081,

China

${ }^{7}$ College of Science and Engineering, Flinders University, GPO Box 2100, Adelaide, South

Australia 5001, Australia

${ }^{8}$ University college of Khurma, Taif University, Taif, 21944, Saudi Arabia

*Correspondence: sajidfiaz50@yahoo.com (S.F.); wangxiukang@ 126.com (X.W.);

Tel.: +92-300-6015-869 (S.F.); +86-911-2332-030 (X.W.)

\begin{abstract}
The hybrid seeds of several important crops with supreme qualities, including yield, biotic and abiotic stress tolerance, have been cultivated from decades. Thus far, a major challenge with hybrid seed, it does not hold ability to produce plants with same qualities over subsequent generations. Apomixis exist naturally an asexual mode of reproduction in flowering plants via avoiding meiosis and ultimately leads to seed production. Apomixis possess potential to preserve hybrid vigor for multiple generations for economically important plant genotypes. The evolution and genetics of asexual seed production is unclear and need much more efforts to find its genetic architecture. To fix hybrid vigor synthetic apomixis has been suggested an alternative. The development of MiMe (Mitosis instead of Meiosis) genotypes are utilized further for clonal gametes production. However, the identification and parental origin of genes responsible for synthetic apomixis are less known and need further understanding. Genome modifications utilizing genome editing technologies (GETs) like clustered regularly interspaced short palindromic repeats (CRISPR)/CRISPR-associated protein 9 (cas9) a reverse genetics tool has paved way to utilize emerging technologies in plant molecular biology. From the last decade, several genes in important crops have been successfully edited. The vast availability of GETs has made the functional genomics studies easy to conduct in crops important for food security. The disruption of expression of genes specific to egg cell MATRILINEAL (MTL) or BABY BOOM1 (BBM1) through CRISPR/Cas genome editing system can promote haploid plants. The establishment of synthetic apomixis by engineering MiMe genotype by genome editing $B B M 1$ expression or disruption of $M T L$ leads toward clonal seed production. In present review, we discussed the current development in plants by utilizing CRISPR/Cas9 technology and its possibility of promoting apomixis in crops
\end{abstract}


to preserve hybrid vigour. In addition to this, genetics, evolution, epigenetic modifications and strategy for MiMe genotype development has been discussed in detail.

Keywords: hybrid vigor, flowering plants, apomixis, CRISPR/Cas9

\section{Introduction}

The scientific, logistical and humanitarian approaches are simultaneously involved to ensure food security. It starts from farmers and breeders, and further extends to policy makers and governments. The universal solution for sustainable food security is difficult owing to differences in cultural values and geographical regions, environments and technologies. However, these challenges are substantial and there is a great potential that needs to be tapped to increase the efficiency and productivity off current agricultural products. The powerful breeding techniques that we have at our disposal are exploiting heterosis in commercially important crops. Hybrid seed production is a successful technology and hybrid seeds produced either by three line or two line system to achieve supreme qualities i.e., premium yield and quality along with resistance against biotic and abiotic stresses, have been cultivated since long time [1]. The two line and three line hybrid development system from which the former is more advantageous than later because in this system photoperiod/thermo-genic male sterile lines have been successfully developed. Moreover, the application of CRISPR has further reduced the time required for hybrid seed development [2]. Recently, a novel male fertility related gene in Wheat $M s l$ has been identified and the biallelic frameshift mutation resulted into complete male sterility that can be utilized for commercial hybrid seed production [3]. Furthermore, the knockout of ZmTMS5 and OsTMS5 in maize and rice, respectively resulted in thermosensitive lines. These lines contain fertile female part and are successfully utilized for hybrid seed production [4]. Moreover, a transgene free maize male sterile line was developed through the targeted mutagenesis of $M S 8$ gene and proved as a valuable source for hybrid development [5] (Figure 1). However, a major challenge with hybrid crops thus far has been that unlike other crops, their progeny segregate and next generation unable to maintain heterosis [6]. These limitations of sexual reproduction create feasibility of apomixis to preserve hybrid seed qualities for multiple generations [7].

Figure 1. Schematic description of the hybrid development utilizing CRISPR/Cas genome editing system. $\mathrm{P} / \mathrm{TGMS}=$ photoperiod/thermo-sensitive genic male sterile.

Apomixis exists naturally and produces offspring, genetically identical to the mother plant. Naturally, the phenomena of apomixis is widespread but exceptional. In 400 families of flowering plants approximately $10 \%$ possesses the phenomena of apomixis which constitutes only $1 \%$ of 40,000 species of those families [8]. Under natural conditions, multiple developmental pathways controlled by various molecular mechanisms play their integral role in achieving apomixis [9]. Apomictic pathways have been categorized as adventitious embryony, a sporophytic type of apomixis, or megaspore mother cell (diplospory) and nearby nucellar cell (apospory) and two gametophytic forms of apomixis. In gametophytic apomixis, a chromosomally unreduced embryo sac develops from the diplospory or from an apospory in a process termed as apomeiosis [10]. Parthenogenesis, the development of the unreduced, unfertilized egg into an embryo, constitutes the second step of the apomictic process [11].

Synthetic apomixis has been proposed by researchers to overcome genetic segregation of $\mathrm{F}_{1}$ hybrids of different crops. The genetic analysis for the inheritance of apomixis in different plant 
species, cereals, grasses and relatives of genera i.e., Tripsacum, Pennisetum, Panicum, Bracchiaria and Paspalum detected a single chromosome segment responsible to induce apomixis [12]. Nevertheless, the efforts to transfer chromosomal segment responsible for the promotion of apomixis remain elusive owing to genetic evolution load [11]. In addition, induced mutations cause complete male or female sterility and seldom production of unreduced gametes [13]. However, the molecular mechanisms controlling apomixis for clonal seed formation are largely unknown and novel plant breeding strategies are desirable to unearth the genetic mechanism controlling such interesting phenomena. If the phenomena of synthetic apomixis introduced in any crop vital for food security, it can helps in fixation and propagation of genotype regardless to genetic complexity in controlling a particular phenotype, ultimately enhancing the application of heterosis fixation in agriculture [14]. Because of this enormous prospective, ingress of apomixis in commercially important crops is considered a hotspot to be studied and explored from plant biologists and seed industry [15]. Wang et al. [16] introduced synthetic apomixis in few fruit crops i.e., citrus and apple however, the epigenetic modifications involvements caused severe seed abortion leading toward the failure in transfer of apomixis in major crops [10]. Recent studies have discussed the genetics and biotechnology approaches [17], integration of knowledge from various aspect of plant breeding [18], and methods for exploiting genome editing, especially CRISPR/Cas genome editing system for improving yield and preservation of heterosis in hybrids [19, 20]. The scientific community equipped with genome editing expertise has played its significant role by transferring relevant and reliable information to beginners in genome editing, on contrary to the proprietary nature of zinc-finger nucleases (ZFNs). In addition, several online platforms are now freely accessible to assist researchers with all their concerns related to genome editing especially CRISPR [21]. Here in present review, we discussed details of apomixis along with the utilization of CRISPR/Cas genome editing system to develop synthetic apomixis as an alternative to preserve hybrid seed vigor in economically important crops.

\section{Evolution of apomixis}

Apomixis is an interesting plant's attribute which allows maternal clones via seed production. Apomixis is an intangible, but revolutionary characteristic for plant breeding and preserving hybrid seed qualities. Recent findings came up with conclusion that apomicts are much useful, with potential to develop more research interest in evolution process of asexual seed production in flowering plants. It is believed among researchers that apomixis alone have had ability to revolutionized the $21^{\text {st }}$ century agriculture in both developed and developing countries. Apomixis has been reported in approximately 35 angiosperm families and in more than 300 plant species [22]. Unfortunately, except few forage grasses and fruit trees, phenomena of apomixis is not obvious in crop species. It was commonly believed that apomixis phenomena mostly occur in polyploidy genotypes [23], however, discovery of apomixis in diploid species [24] abolished the necessity of polyploidy for apomixis [25]. The four perennials in nature apomictic Cenchrus spp. i.e., C. myosuroides an obligate sexual, diploid apomictic $C$. setigerus, C. echinatus and $C$. myosuroides polyploidy and sexual in nature indicating apomixis without polyploidy. Thus, there is still quest for in-depth research to challenge the strong but less understood correlation of polyploidy and apomixis. Moreover, the faithful transmission of maternal genotypes to the next generations also need sufficient scientific understanding due to genetic variability among apomictic populations. There are at least three proposed mechanisms that may cause genetic variability in apomictic plants or species [26]. First, the accumulation of new mutations [22] second, the involvement of irregular sexual reproduction causing recombinant genotypes in 
populations often called leaky apomixis [27] and third, the facultative mode of reproduction [28]. Many researchers around the globe have detailed analyzed and discussed these mechanism [29] (Figure 2).

Figure 3. The emergence of apomictic types. (a) Amphimixis (b) Facultative apomixis and (c) Obligate apomixis [30].

\section{Genetics and Genomics of Apomixis}

Apomixis is qualitative in nature and involves components of genetic analysis. The molecular studies to unearth genetic basis for apomixis are unable to explain mysterious phenomena, because most of the apomicts do not exist in important crops [31]. There is well established association between polyploidy and gametophytic apomixis [32]. The discovery of apomixis phenomena in diploid plant populations disassociate the absolute necessity of polyploidy for apomixis [25]. However, some researchers suggested the genetic control of apomixis is not an independent trait but triggered by the epigenetic temporal and spatial modification of the sexual system [33]. Earlier, it was thought the genetic control of apomixis controlled by recessive genes and their balance may change after each successful crossing. Recently, a new concept for inheritance process predicted the involvement of a single major regulatory gene or a set of key dominant genes, allowing megaspore mother cell or somatic nuclear cell to form an embryo sac and an embryo from unreduced egg cell without fertilization $[32,34]$. Whereas, the spatial and temporal distribution of these genes during developmental process is still not well understood. The genetic mechanism of asexual reproduction is complicated in nature therefore, inter-specific hybrids or intra-specific hybrids within agamic complex are widely studied for the segregation (apomeiosis, parthenogenesis and functional endosperm development) of apomixis. Moreover, the phenotypic analysis of plant generations reproduction either asexually or sexually involves time consuming cytology or progeny testing. A single gene with regulatory function was initially proposed for promoting asexual reproduction. Recently, Albertini et al. [31] has reported three key components (apomeiotic megaspores, parthenogenic unreduced egg cells, and modified endosperms) of gametophytic apomixis depends on three independent mendelian loci of major influence. The relationship between rich retrotransposon regions of heterochromatin and apomictic mechanisms has elevated possibility for the role of DNA and/or RNA interference in regulating expression of apomictic genes [35]. Some researchers believe the influence of specific genes encoding novel proteins for new functions which are absent from plant with sexual reproduction and ultimately identification of candidate genes/loci. Some genes originally fine mapped and characterized in or from species reproduce sexually may play an important role in promoting apomixis through loss of function or change in function of genes differentiating between apomictic and sexual pathways. However, genetic factors controlling apomixis also contain association with some lethal genes influencing normal functioning of both male and female gametes which need further investigation.

The apomictic species contains suppressed recombination however, the genes controlling the various components of apomixis are identified and sequencing of these genes further enhance understanding toward their potential utilization. The random amplified polymorphic DNA based analysis helped in the identification of apospory-specific genomic region (ASGR) of Pennisetum both in sexual and apomictic plants [36]. The bacterial artificial chromosome (BAC) clones sequencing of $A S G R$ from Pennisetum and Cenchrus revealed 40 putative protein coding regions, two of them showed sequence similarity to $B A B Y B O O M(B B M)$ gene [37]. The $B B M$ gene was identified in Brassica napus as AP2-domain transcription factor, overexpression analysis in 
arabidopsis showed its involvement in embryo development from vegetative tissues [38]. The $A S G R-B B M$ possesses potential and are candidate genes for induction and/or maintenance of apomictic events [37]. The amplified fragment length polymorphism (AFLP) markers screening in apomictic and sexual plants of Hypericum lead toward identification of locus HYPERICUM APOSPORY. The perfect co-segregation of AFLP further utilized to screen the BAC library of Hypercium. The screening helped in identification of a single clone containing aubiquitinmediated E3 ligase [39]. This ARIADNE 7-like E3 ligase (HpARI) has been proposed candidate for the HAPPY locus, as one of the four HPARI alleles within the tetraploid apomict is truncated compared to sexual plants. The HPARI act as negative regulator and interact with rest of three alleles. The E3 ligase is responsible for ubiquitin-mediated protein degradation involved in embryo sac development. In mitotic spindle function and nuclear fate in developing maize embryo sac MATH-BTB protein MAB interacts with an E3 ubiquitin ligase component (Cullin3a) [40]. Any modification in expression or function of $H p A R I$ E3 ligase may impact embryo sac development and apomixis events in Hypericum.

The spatial and temporal alternation in expression of sexual pathway controlling genes may cause apomixis therefore, the comparative gene expression analysis may provide opportunity to identify such genes. The low depth express sequence tag (EST) libraries consist of apomictic and sexually propagated aposporous grass Brachiaria brizantha revealed sequence similarity of genes involved in female embryo sac development [41]. The characterization of identified genes mainly expressed in aposporous initial cells appear. The helicase BbrizHelic, BbrizAGL6 transcription factor and BbrizStil stress inducing protein play integral role in aposporous initial cells [42]. In Poa pratensis, cDNA-AFLP helped in identification and characterization of two candidate genes; SOMATIC EMBRYOGENESIS RECEPTOR-LIKE KINASE (PpSERK) and APOSTART [43]. Both PpSERK and APOSTART genes were isolated in two copies, there are several alleles present within $P$. pratensis genome. The PpSERK gene is tyrosine kinase that switch aposporous initial cell to form and develop embryo sac in somatic cells [43]. APOSTART contains a lipid binding START domain and is believed to have a role in meiosis. APOSTART may also be related to program cell death and the degeneration of the nonfunctional megaspores, with one of the two isolated copies (APOSTART1) overexpressed in sexual lines relative to apomicts. The Arabidopsis APOSTART1 ortholog is expressed in mature female embryo sacs and developing embryos, and the phenotype of APOSTART1/APOSTART2 double mutants suggests that this gene has a role in embryo and seed development [11]. There are several other genes identifies by several researchers playing their important role in controlling asexual reproduction and can be utilized in future to understand their regulatory functions (Table 1).

\section{Epigenetic control of apomixis}

The variable developmental processes and the cells involved in apomixis mechanism indicates the influence of epigenetic regulatory mechanism in controlling apomixis process. To investigate the hypothesis cytosine methylation, histone modification and comparative analysis of small-RNA has been undertaken both in apomictic and sexually propagated plants. In higher plants the activation or movement of transposable elements (TEs) covers a significant proportion of genome influencing evolution of genome, alternation in gene expression and frame shift mutations [44]. To keep intact the genomic integrity of host genome TEs movement is important to be regulated. The centromeric or telemetric regions are generally saturated with TEs with high rate of methylation and packed as heterochromatic regions. In maize, it has been proved that in heterochromatin regions TEs are specific for methylation indication their integral role in 
alternation of structural and functional properties in plants. It has also been speculated the role of retrotransposons in apomictic development however, it needs further proof of concept from both apomictic and sexually propagated plants. From the Cenchrus ciliaris public database of EST/BAC sequence several classes of retrotransposons were identified and possible role in apomixis was elucidated. Based on expression analysis from 19 identified retrotransposons six were found associated with apomictic activity. Among six retrotransposons, the C-105 was further utilized to understand epigenetic regulation based on differential activity in both apomictic and sexual propagated plants of $C$. ciliaris. The bisulfite sequencing of retrotransposon $(\sim 0.3 \mathrm{~Kb}$ fragment) shown $95 \%$ methylation of cytosine in sexually propagated plant as compared to apomictic. The reversible ability of epigenetic modification in sexual pathways is also hypothesized. The analysis of Arabidopsis mutant ovule epigenetically controlled by sRNA-mediated silencing pathway involving ARGONAUTE 9 (AGO9) protein showed transition from apomictic to sexual reproduction [45]. The loss of function of mutant protein ago104 homologue to maize AGO9 shown apomixis like characteristic, with $\sim 70 \%$ of functional female gametes. The comparative analysis of sexual reproduction in maize with its apomictic wild relative Tripsacum further supported the epigenetic regulation of apomixis. A small number of chromatin enzymes e.g., CMT3 and DRM2 shown difference of expression among apomictic and sexual plants. Moreover, it has been recently revealed involvement of mutant alleles of genes responsible for DNA methylation and siRNA pathways in of apospory or diplospory of arabidopsis and maize. However, there is still quest to understand the underline mechanism linking epigenetic modification with establishment of apomixis in plants. Thus, the underlying mechanism/theory controlling epigenetic regulation of apomixis is worth to study especially its ability to revert sexual propagation from apomictic mode [22]. The genetics, genomics and epigenetic modification to promote apomixis in flowering plants is well studies whereas, the perspective of induced apomixis through the genome editing technique i.e., CRISPR/Cas system is at foundation stage as only few reports have been published. The reports indicated the potential role of engineered apomixis to preserve hybrid seed vigor which will ultimately lower the cost of farmer to buy hybrid seed every year.

\section{Synthetic apomixis through MiMe strategy}

There are three most common approaches in plant breeding to convert crop plants from sexual to apomictic mode of reproduction; i) wide crosses with apomictic wild relatives, ii) mutation breeding, and iii) genetic transformation techniques. Sexual hybridization to transfer apomixis through interspecific hybridization rely heavily on the availability of wild relatives. In maize the apomixis was transferred from its wild relative Tripsacum through hybridization whereas, the produced hybrid after series of backcrosses was sterile in nature and facultative apomicts were not produced to recover maize genome [46]. So far, it is generally believed the transfer of apomixis through wide crosses is not a successful approach. Meanwhile, the application of artificial mutation studies has provided significant evidences regarding genetic architecture of apomixis. The meiosis and mitosis are distinguished on the basis of three integral characteristics. Firstly, the induced DNA double standard breaks (DSBs) with recombination and pairing between homologous chromosomes. Secondly, the first cell division leads towards monopolar orientation of kinetochores of sister chromatids. Thirdly, after genome duplication the division of cell took place two times [47]. The genetic factors controlling these three developmental process have been identified in plants. The genes SPO11-1, SPO11-2, HOMOLOGOUS PAIRING ABERRATION IN RICE MEIOSIS1 (PAIR1), PUTATIVE RECOMBINATION INITIATION DEFECT1 (PRD1), PUTATIVE RECOMBINATION INITIATION DEFECT2 (PRD2), MEIOTIC TOPOISOMERASE 
VIB-LIKE (MTOPVIB), DSB FORMATION (DFO), CENTRAL REGION COMPONENT 1 $(C R C 1)$, and $P 31^{\text {comet }}$ are indispensable for induction of DSBs in plants. The induced mutation in above mentioned genes disrupt the normal pairings and recombination of homologous chromosomes [48]. The monopolar orientation of kinetochores during first meiotic division is mainly regulated by meiotic cohesion complex controlled by a major component REC8 [49]. The genes OMISSION OF SECOND DIVISION (OSD1) and TARDY ASYNCHRONOUS MEIOSIS (TAM) control entry into the second division of meiosis. The mutation in any of these genes impact the second division leading toward production of diploid gametes in both male and female (d'Erfurth et al., 2010). It has been observed double mutant spol1-1 and rec8 produced a mitoticlike first meiotic division [50]. The osd1, rec8 and spol1-1 triple mutants or tam, rec8 and spol11 triple mutants exhibited apomeiosis phenotypes in which meiosis switched into mitosis-like division and genotype was called MiMe [47]. The MiMe strategy helped in development of viable diploid gametes and successfully transferred into rice by combining the mutations of the genes (PAIR1, REC8, and OSD1) involved in the same process, suggesting that this strategy could be widely applied in flowering plants [8]. The synthetic apomixis can be achieved through eliminating one set of chromosomes from either parent has to be eliminated. Centromeres are the loci where the microtubule of the spindle are located and are essential for chromosome segregation during cell division. Centromeres are specified by a centromere-specific histone 3 (CENH3) protein in plants. The engineered genome elimination line (GEM) can be achieved through the application of CENH3 and further crossed with wild type plants. The clonal seeds were generated through crossing MiMe plants with GEM line and ultimately open plethora of option for the clonal reproduction through seeds similar to the apomixis [51]. Nonomura et al. [52] reported an ortholog $A G O 5$ in rice which proved to play an integral role for pre-meiotic mitosis and meiosis similarly, in maize the lacking of ortholog AGO9 leads toward the production of viable gametes without meiosis [53]. In Arabidopsis, the screening of parallel mutants for apomixis helped in identification of genes responsible for initiation of fertilization-independent seed (FIS) development. These FIS genes encode protein which resemble with members of polycomb related complex [54]. The mutants of FIS genes are known to trigger endosperm development through asexual mode of reproduction up to varying extent. Whereas, the embryo initiation for most of fis mutants is either low or does not took place. However, the epistatic effect of genes rendering natural process of seed development believed a limitation to get apomictic plants. So far, several genes and their mutants have been developed promoting phenomena of apomixis in various plants species and are well reviewed by $[10,11]$. The genetic transformation approach contains much potential but the limited genetic information is the only limiting factor. Map based cloning for genes controlling apomixis in crop plants is much slow owing to suppressed recombinants and excess repeated DNA sequences in apomixis associated genomic regions. Whereas, in few plant species the apomictic genes were detected utilizing deletion studies [55]. Recently, another approach called "conditional apomixis" has gained the scientific ground which switched default mode of plant reproduction system to apomictic or sexual mode of reproduction [56]. Conditional apomixis in plants can be achieved utilizing special promoters of which expression can be altered by certain chemicals or through epigenetic factors [35]. However, the classical methods in plant breeding are time consuming, labor intensive, costly, less reliable and less flexible. Due to these limitations in classical breeding approaches the modern genome editing technologies have gain the scientific ground and are widely utilized by plant breeders to meet their objective with more ease and precision. 


\section{Synthetic apomixis through genome editing to preserve hybrid vigor}

In modern time, CRISPR technology is widely utilized with tremendous success in agriculture especially for crops essential for food security perspectives i.e., rice, wheat, maize, Arabidopsis, cotton, tomato and potato. The user friendly and low budget requirement of genome editing has spurred the research for crop-trait development not only in academia but also with private companies dealing in agriculture products [57]. Moreover, the genome editing technology has not only speed up the breeding effort to improve yield, grain quality, resistance against a/biotic stresses and domestication in plants, but also withstand in diverse environment with high value traits such as hybrid vigour or heterosis, which has proved to be a major challenge for researchers and farmers. It is a significantly desirable goal that could change the agriculture production system. The de novo targeted modification can alter the sexual mode of reproduction to apomixis and has been successfully employed in Arabidopsis [51]. The BBM gene belongs to the APETALA 2/ETHYLENE RESPONSE FACTOR (AP2/ERF) DNA-binding domain family and is expressed in sperm cells. The $A P 2 / E R F$ gene family is divided into $E R F$-like, which has a single $A P 2$ domain, and $A P 2$-like, which contains two AP2 domains (repeat 1 and 2) that are similar to each other and separated by a linker region. In Pennisetum squamulatum, apomixis is transmitted by a physically large, hemizygous, non-recombining chromosomal region, the ASGR [12]. Several copies of PsASGR-BBML genes are considered candidate genes promoting parthenogenesis due to their strong linkage between apomictic Pennisetum/Cenchrus species [37]. Conner et al., [58], reported a mutant of CcASGR-BBML gene in Cenchrus ciliaris which ultimately lost its ability to experience parthenogenesis moreover, the ASGR-BBML are in Arabidopsis and Brassica [38]. Conner et al., [9], successfully reduced the expression of $P S A S G R-B B M L$ in apomictic $\mathrm{F}_{1}$ RNAi transgenic plants and endorse the key role of $P S A S G R-B B M L$ to promote parthenogenesis in Pennisetum squamulatum which can further lead toward haploid induction to vigorously obtain homozygous transgenic lines for breeding. Khanday et al. [7] triple knockout of the genes $B B M 1$, $B B M 2$ and $B B M 3$ caused embryo arrest and abortion, which are fully rescued by male-transmitted $B B M 1$. These findings suggest that the requirement for fertilization in embryogenesis is mediated by male genome transmission of pluripotency factors. When genome editing to substitute the mitosis for meiosis $(M i M e)$ phenotype $[8,47]$ is combined with the expression of $B B M 1$ in the egg cell, clonal progeny can be obtained that retain genome-wide parental heterozygosity. The synthetic asexual-propagation trait is heritable through multiple generations of clones. Moreover, [14] multiplex editing of three (REC8, PAIRI and OSD1) key meiotic genes in hybrid rice leads to the production of clonal diploid gametes and tetraploid seeds. Next, editing of the $M T L[4,59]$ gene involved in fertilization results in the induction of haploid seeds in hybrid rice. By simultaneous editing of these four endogenous genes in hybrid rice using the CRISPR/Cas9 system, plants able to propagate clonally through seeds were obtained. The quadruple AOP (Apomictic Offspring Producer) mutants obtained through the knockout of OsSPO11-1, OsREC8, OSOSD1 and OsMATL produced the MiMe phenotype. The mutant has the ability to produce apomictic plants [20]. However, thus far, the number of viable clone seeds with their original hybrid genetics intact are much lower than expected and this area needs further research. (Figure 3).

Figure 4. An illustration of promoting apomixis for hybrid vigour preservation through the CRISPR/Cas9 system. Hybrid development took place via genome editing of genes or through conventional breeding techniques. To fix hybrid vigor in $\mathrm{F}_{2}$ scientist are promoting apomixis in hybrid seed to keep intact the characteristics of $\mathrm{F}_{1}$ hybrid seed. The MiMe phenotypes is achieved 
through disruption of gene controlling meiosis II which leads towards unreduced embryo sac essential for apomeiosis. However, in other case the hybrid segregate in $\mathrm{F}_{2}$ plant populations and all the important characteristics are lost.

The only drawback with hybrid technology comprised of hybrid vigour along with other desired traits lost due to genetic segregations in subsequent generation. Moreover, the cost of seed and new seed for every sowing are the big challenges faced by subsistence farmers [60]. The preservation of hybrid seed qualities had significant implication to ensure food security, environmental preservation and employment. Ensuring that crops pass on hybrid qualities to seeds has been a major challenge, but researchers equipped with modern plant breeding technologies are striving to overcome this challenge. The findings in above mentioned studies revealed a straight forward strategy to promote apomixis to preserve hybrid vigour in rice. Moreover, the approach can also work for other cereal crops that have equivalent genes, such as wheat, corn, barley and millets, by increasing the percentage of seeds with the same hybrid vigour, and ultimately, these seeds can reach farmers' fields. Moreover, identifying genes controlling the molecular mechanism of apomictic reproduction is critical to understand genetics architecture, which is still less understood.

\section{Limitations to synthetic apomixis}

The utilization of CRISP/Cas system for synthetic apomixis has been successfully employed in rice, however, the seed production with intact hybrid vigor was significantly reduced. Wang et al., [14], reported the MiMe does not significantly reduced the seed production whereas, the mutation in MTL leads toward haploid induction at expense of seed production in rice. Similarly, in Maize the large scale chromosome fragmentation caused reduction in fertility owing to post-meiosis in the $m t l$ gametophyte [4]. The fragmentation leads toward the imbalance between genome of both maternal and paternal side. There are several genes investigated in Arabidopsis and apomictic plants involved in embryo and endosperm development. The novel strategy i.e., combining Fix strategy with genes promoting auto-endosperm development may resolve the issue of low fertility caused by Fix strategy. However, the Fix strategy can be successfully employed in crops where seed production is secondary objective e.g., vegetables and forage crops which also need further investigation. Hand and Kultunow, [10], reported that engineered Fix strategy is not much effective as naturally occurring apomixis because of penetration ability. Therefore, the identification of novel genes controlling apomixis need to explore which ultimately helps to increase clonal seeds. On contrary, techniques to distinguish clonal seeds or plants from offspring could be developed to further expand the application of Fix strategy in agriculture. The mechanism of heterosis is less known meanwhile, it was generally believed degree of genetic distance contribute toward heterosis [61]. However, the recent studies have displayed the involvement of epigenetic also contribute their part in heterosis [62]. Wang et al., [14], re-sequenced the whole genome of parent plants and clonal plants and reported the faithful transmission of genetic information which demonstrated that epigenetic information also transferred normally across generation. However, the epigenetic modification cannot be ruled out but modifications were too minor to induce detectable phenotypic changes in rice field. Further studies are thus required in several other crops to investigate the phenotypic stability over multiple generations.

\section{Conclusion and future perspectives}


The phenomena of apomixis remain challenging for the plant geneticists however, its potential benefits remain focus of enormous interest for plant breeders. Both the naturally existing genes and induced mutation can divert the natural sexual pathway to apomixis which can be further investigated. A combined strategy which can target apomeiosis, parthenogenesis, and seed formation might be utmost important to exploit full potential of apomixis eliminating breeding barriers. It has been historically proved that development of hybrids for different crops has helped farmers to obtain benefits, such as maximum crop yield, grain quality, crops with increased resistance to biotic and abiotic stresses, but the hybrid seed lacks in ability to produce plants with the same qualities in subsequent generations. Hence, farmers have had no option rather than to buy expensive hybrid seeds every year. The identification of QTLs/genes through new mapping technologies, that is, comparative mapping, linkage disequilibrium mapping, deletion mapping and new high-throughput sequencing methods will help to penetrate in the core of apomixis chromosomal regions. Similarly, high-throughput technologies can expose or remove the evolutionary genetic load of genes and epigenetic modifications related to apomixis and can be further utilized in agriculture as a tool to fix elite genotypes important for food security. The successful application of genome editing technology for targeted mutagenesis has opened further avenues of research for understanding molecular mechanisms controlling apomixis. The efficiency of clonal propagation in crops, particularly in rice, has been limited by the frequency of parthenogenesis. However, it could be improved in future through studying different promoters of genes or incorporating specific alleles that exhibit full or partial dominance.

9. Author Contributions: SF and XW conceived the idea and drafted the manuscript. SF, XW, and AR collected the literature review. BA and RM provided with technical assistance. AR and SA provided the illustration and formatted the manuscript. SF and SA helped in final editing of the manuscript. All authors listed have made substantial, direct, intellectual contributions to the work and have approved it for publication.

10. Funding: The publication of the present work is supported by the National Key Research and Development Program of China (grant no. 2017YFC0504704) and the National Natural Science Foundation of China (51669034, 41761068, 51809224).

11. Acknowledgments: The authors are thankful to Mehmood Ali Noor for providing an insights to further improve the manuscript.

12. Conflicts of Interest: The authors have no competing interest to declare. 


\section{References}

1. Sheng, Z.; Wei, X.; Shao, G.; Chen, M.; Song, J.; Tang, S.; Luo, J.; Hu, Y.; Hu, P.; Chen, L. Genetic analysis and fine mapping of $t m s 9$, a novel thermosensitive genic male-sterile gene in rice (Oryza sativa L.). Plant Breed. 2013, 132, 159-164.

2. Barman, H. N.; Sheng, Z.; Fiaz, S.; Zhong, M.; Wu, Y.; Cai, Y.; Wang, W.; Jiao, G.; Tang, S.; Wei, X. Generation of a new thermo-sensitive genic male sterile rice line by targeted mutagenesis of TMS5 gene through CRISPR/Cas9 system. BMC Plant Biol. 2019, 19, 109.

3. Okada, A.; Arndell, T.; Borisjuk, N.; Sharma, N.; Watson, H.N.S.; Tucker, E. J.; Baumann, U.; Langridge, P.; Whitford, R. CRISPR/Cas9-mediated knockout of Ms 1 enables the rapid generation of male-sterile hexaploid wheat lines for use in hybrid seed production. Plant Biotechnol. J. 2019, 17, 1905-1913.

4. Li, X.; Meng, D.; Chen, S.; Luo, H.; Zhang, Q.; Jin, W.; Yan, J. Single nucleus sequencing reveals spermatid chromosome fragmentation as a possible cause of maize haploid induction. Nat. Commun. 2017, 8, 991.

5. Chen, R.; Xu, Q.; Liu, Y.; Zhang, J.; Ren, D.; Wang, G.; Liu, Y. Generation of Transgene-Free Maize Male Sterile Lines Using the CRISPR/Cas9 System. Front. Plant Sci. 2018, 9, 1180

6. Huang, X.; Yang, S.; Gong, J.; Zhao, Q.; Feng, Q.; Zhan, Q.; Zhao, Y.; Li, W.; Cheng, B.; Xia, J. Genomic architecture of heterosis for yield traits in rice. Nature. 2016, 537, 629-633.

7. Khanday, I.; Skinner, D.; Yang, B.; Mercier, R.; Sundaresan, V. A male-expressed rice embryogenic trigger redirected for asexual propagation through seeds. Nature. 2019, 565, 9195.

8. Mieulet, D.; Jolivet, S.; Rivard, M.; Cromer, L.; Vernet, A.; Mayonove, P.; Pereira, L.; Droc, G.; Courtois, B.; Guiderdoni, E. Turning rice meiosis into mitosis. Cell Res. 2016, 26, 12421254.

9. Conner, J. A.; Mookkan, M.; Huo, H.; Chae, K.; Ozias-Akins, P. A parthenogenesis gene of apomict origin elicits embryo formation from unfertilized eggs in a sexual plant. Proc. Natl. Acad. Sci. 2015, 112, 11205-11210.

10. Hand, M.L.; Koltunow, A.M. The genetic control of apomixis: asexual seed formation. Genet. 2014, 197, 441-450.

11. Barcaccia, G.; Albertini, E. Apomixis in plant reproduction: a novel perspective on an old dilemma. Plant Reprod. 2013, 26, 159-179.

12. Ozias-Akins, P.; van Dijk, P.J. Mendelian genetics of apomixis in plants. Annu. Rev. Genet. 2007, 41, 509-537.

13. Ravi, M.; Marimuthu, M.P.; Siddiqi, I. Gamete formation without meiosis in Arabidopsis. Nature. 2008, 451, 1121.

14. Wang, K. Fixation of hybrid vigor in rice: synthetic apomixis generated by genome editing. aBIOTECH 2019, 1-6.

15. Sailer, C.; Schmid, B.; Grossniklaus, U. Apomixis allows the transgenerational fixation of phenotypes in hybrid plants. Curr. Bio. 2016, 26, 331-337.

16. Wang, X.; Yuantao, X.; Zhang, S.; Cao, L.; Hunag, Y.; Cheng, J.; Wu, G.; Tian, S.; Chen, C.; Liu, Y.; et al. Genomic analyses of primitive, wild and cultivated citrus provide insights into asexual reproduction. Nat. Genet. 2017, 49, 765.

17. Yang, Y.; Zhu, G.; Li, R.; Yan, S.; Fu, D.; Zhu, B.; Tian, H.; Luo, Y.; Zhu, H . The RNA editing factor SIORRM4 is required for normal fruit ripening in tomato. Plant physiol. 2017. $175,1690-1702$. 
18. Sun, Y.; Jiao, G.; Liu, Z.; Zhang, X.; Li, J.; Guo, X.; Du, W.; Du, J.; Francis, F.; Zhao, Y.; Xia, L. Generation of high amylose rice through CRISPR/Cas9-mediated targeted mutagenesis of starch branching enzymes. Front. Plant Sci. 2017. 8, 298.

19. Li, M.; Li, X.; Zhou, Z.; Wu, P.; Fang, M.; Pan, X.; Lin, Q.; Luo, W.; Wu, G.; Li, H. Reassessment of the four yield-related genes Gnla, DEP1, GS3, and IPA1 in rice using a CRISPR/Cas9 system. Front. Plant Sci. 2016. 7, 377.

20. Xie, E.; Li, Y.; Tang, D.; Lv, Yanli.; Shen, Y.; Cheng, Z. A strategy for generating rice apomixis by gene editing. J. Integr. Plant Biol. 2019. 61, 911-916.

21. Xu, R.; Yang, Y.; Qin, R.; Li, H.; Qiu, C.; Li, L.; Wei, P.; Yang, J.; Rapid improvement of grain weight via highly efficient CRISPR/Cas9-mediated multiplex genome editing in rice. Journal of genetics and genomics. 2016. 43, 529-532.

22. Kumar, S. Epigenetic control of apomixis: a new perspective of an old enigma. Adv. Plants Agric. Res. 2017. 7, 10.15406.

23. Carman, J.G. Asynchronous expression of duplicate genes in angiosperms may cause apomixis, bispory, tetraspory, and polyembryony. Biol. J. Linn. Soc. 1997. 61, 51-94.

24. Voigt-Zielinski, M.L.; Piwczyński, M.; Sharbel, T.F. Differential effects of polyploidy and diploidy on fitness of apomictic Boechera. Sex. Plant Reprod., 2012. 25, 97-109.

25. Rodriguez-Leal, D.; Vielle-Calzada, J.P. Regulation of apomixis: learning from sexual experience. Curr. Opin. Plant Biol. 2012. 15, 549-555.

26. Adolfsson, S.; Bengtsson, B.O. The spread of apomixis and its effect on resident genetic variation. J. Evol. Biol. 2007. 20, 1933-1940.

27. Balloux, F.; Lehmann, L.; de Meeûs, T. The population genetics of clonal and partially clonal diploids. Genetics 2003. 164, 1635-1644.

28. Bengtsson, B.O. Genetic variation in organisms with sexual and asexual reproduction. J. Evol. Biol. 2003. 16, 189-199.

29. Yadav, C.B.; Suresh, Y.Q.; Kumar, M.G.; Bhat, G.V. Genetic linkage maps of the chromosomal regions associated with apomictic and sexual modes of reproduction in Cenchrus ciliaris. Mol. Breed. 2012. 30, 239-250.

30. Fei, X.; Shi, J.; Liu, Y.; Niu, J.; Wei, A. The steps from sexual reproduction to apomixis. Planta 2019. 249, 1715-1730.

31. Albertini, E.; Barcaccia, G.; Mazzucato, A.; Sharbel, T.F.; Falcinelli, M. Apomixis in the era of biotechnology. In Plant developmental biology, Biotechnological perspectives. Springer 2010. 405-436.

32. Savidan, Y. Apomixis: genetics and breeding. Plant Breed. Rev. 2000. 18, 13-86.

33. Grimanelli, D. Epigenetic regulation of reproductive development and the emergence of apomixis in angiosperms. Curr. Opi. Plant Biol. 2012. 15, 57-62.

34. Grossniklaus, U.; Spillane, C.; Page, D.R.; Kohler, C. Genomic imprinting and seed development: endosperm formation with and without sex. Curr. Opi. Plant Biol. 2001. 4, 2127.

35. Pupilli, F.; Barcaccia, G. Cloning plants by seeds: inheritance models and candidate genes to increase fundamental knowledge for engineering apomixis in sexual crops. J. Biotech. 2012. 159, 291-311.

36. Ozias-Akins, P.; Roche, D.; Hanna, W.W. Tight clustering and hemizygosity of apomixislinked molecular markers in Pennisetum squamulatum implies genetic control of apospory by a divergent locus that may have no allelic form in sexual genotypes. Proc. Natl. Acad. Sci. 1998. $95,5127-5132$. 
37. Conner, J.A.; Goel, S.; Gunawan, G.; Cordonnier-Pratt, M.M.; Johnson, V. E.; Liang, Chun.; Wang, H.; Pratt, L. H.; Mullet. J.E.; DeBarry, J.; et al. Sequence analysis of bacterial artificial chromosome clones from the apospory-specific genomic region of Pennisetum and Cenchrus. Plant Physiol. 2008. 147, 1396-1411.

38. Boutilier, K.; Offringa, R.; Sharma, V.K.; Kieft, H.; Ouellet, T.; Zhang, L.; Hattori, J.; Liu C.M.; van Lammeren, A.A.; Miki, B.L.; et al. Ectopic expression of BABY BOOM triggers a conversion from vegetative to embryonic growth. Plant Cell. 2002. 14, 1737-1749.

39. Schallau, A.; Arzenton, F.; Johnston, A.J.; Hähnel, U.; Koszegi, D.; Blattner, F.R.; Altschmied, L.; Haberer, G.; Barcaccia, G.; Bäumlein, H. Identification and genetic analysis of the APOSPORY locus in Hypericum perforatum L. Plant J. 2010. 62, 773-784.

40. Juranić, M.; Srilunchang, K.; Krohn, N.G.; Levanić, D.L.; Sprunck, S.; Dresselhaus, T. Germline-specific MATH-BTB substrate adaptor MAB1 regulates spindle length and nuclei identity in maize. Plant Cell, 2012. 24, 4974-4991.

41. Silveira, E.D.; Guimarães,L.A.; de Alencar Dusi, D.M.; da Silva F.R.; Martins, N.F.; do Carmo Costa, M.M.; Alves-Ferreira, M.; de Campos Carneiro, V.T. Expressed sequence-tag analysis of ovaries of Brachiaria brizantha reveals genes associated with the early steps of embryo sac differentiation of apomictic plants. Plant Cell Rep. 2012. 31, 403-416.

42. Guimarães, L.A.; Dusi, D.M.A.; Masiero, S.; Resentini, F.; Gomes, A.C.M.M. BbrizAGL6 is differentially expressed during embryo sac formation of apomictic and sexual Brachiaria brizantha plants. Plant Mol. Biol. Rep. 2013. 31, 1397-1406.

43. Albertini, E.; Marconi, G.; Reale, L.; Barcaccia, G.; Porceddu, A.; Ferranti, F.; Falcinelli, M. SERK and APOSTART. Candidate genes for apomixis in Poa pratensis. Plant Physiol. 2005. 138, 2185-2199.

44. Yan, H.; Kikuchi, S.; Neumann, P.; Zhang, W.; Wu, Y.; Chen, F.; Jiang, J. Genome-wide mapping of cytosine methylation revealed dynamic DNA methylation patterns associated with genes and centromeres in rice. Plant J. 2010. 63, 353-365.

45. Law, J.A.; Jacobsen, S.E. Establishing, maintaining and modifying DNA methylation patterns in plants and animals. Nat. Rev. Gen. 2010. 11, 204.

46. Kandemir, N.; Saygili, I. Apomixis: new horizons in plant breeding. Turk. J. Agri. For. 2015. 39, 549-556.

47. d'Erfurth, I.; Jolivet, S.; Froger, N.; Catrice, O.; Novatchkova, M.; Mercier, R.; Turning meiosis into mitosis. PLoS Biol. 2009. 7, e1000124.

48. Tang, Y.; Yin, Z.; Zeng, Y.; Zhang, Q.; Chen, L.; He, Y.; Lu, P.; Ye, De.; Zhang, X.; MTOPVIB interacts with AtPRDI and plays important roles in formation of meiotic DNA double-strand breaks in Arabidopsis. Sci. Rep. 2017. 7, 10007.

49. Shao, T.; Tang, D.; Wang, K.; Wang, M.; Che, L.; Qin, B.; Yu, H.; Li, M.; Gu, M.; Cheng, Z. OsREC8 is essential for chromatid cohesion and metaphase I monopolar orientation in rice meiosis. Plant Physiol. 2011. 156, 1386-1396.

50. Chelysheva, L.; Diallo, S.; Vezon, D.; Gendrot, G.; Vrielynck, N.; Belcram, K.; Rocques, N.; Márquez-Lema, A.; Bhatt, A.M.; Horlow, C. AtREC8 and AtSCC3 are essential to the monopolar orientation of the kinetochores during meiosis. J. Cell Sci. 2005. 118, 4621-4632.

51. Marimuthu, M.P.; Jolivet, S.; Ravi, M.; Pereira, L.; Davda, J.N.; Cromer, L.; Wang, L.; Nogué, F.; Chan, S.W.L.; Siddiq, I. Synthetic clonal reproduction through seeds. Sci. 2011. 331, 876876. 
52. Nonomura, K.I.; Morohoshi, K.; Nakano, M.; Eiguchi, M.; Miyao,A.; Hirochika, H.; Kurata, $\mathrm{N}$. A germ cell-specific gene of the ARGONAUTE family is essential for the progression of premeiotic mitosis and meiosis during sporogenesis in rice. Plant Cell 2007. 19, 2583-2594.

53. Singh, M.; Goel, S.; Meeley, R.B.; Dantec, C.; Parrinello, H.; Michaud, C.; Leblanc, O.; Grimanelli, D. Production of viable gametes without meiosis in maize deficient for an ARGONAUTE protein. Plant Cell 2011. 23, 443-458.

54. Koltunow, A.M.; Grossniklaus, U. Apomixis: a developmental perspective. Annu. Rev. Plant Biol., 2003. 54, 547-574.

55. Kotani, Y.; Henderson, S. T.; Suzuki, G.; Johnson, S. D.; Okada, T.; Siddons, H.; Mukai, Y.; Koltunow, A. M. The loss of apomeiosis (LOA) locus in Hieracium praealtum can function independently of the associated large-scale repetitive chromosomal structure. New Phytol. 2014, 201, 973-981.

56. Spillane, C.; Curtis, M.D.; Grossniklaus, U. Apomixis technology development virgin births in farmers' fields? Nat. Biotech. 2004. 22, 687.

57. Fiaz, S.; Ahmad, S.; Noor, M.A.; Wang, X.; Younas, A.; Riaz, A.; Riaz, A.; Ali, F. Applications of the CRISPR/Cas9 system for rice grain quality improvement: Perspectives and opportunities. Inter.J Mol. Sci., 2019. 20, 888.

58. Conner, J.A.; Gunawan, G. ; Ozias-Akins, P. Recombination within the apospory specific genomic region leads to the uncoupling of apomixis components in Cenchrus ciliaris. Planta, 2013. 238, 51-63.

59. Kelliher, T.; Starr, D.; Richbourg, L.; Chintamanani, S.; Delzer, B.; Nuccio, ML.; Green, J.; Chen, Z.; McCuiston, J.; Wang, W. MATRILINEAL, a sperm-specific phospholipase, triggers maize haploid induction. Nature 2017. 542, 105.

60. Janaiah, A.; Hossain, M.; Husain, M. Hybrid rice for tomorrow's food security: can the chinese miracle be replicated in other countries? Outlook Agri. 2002. 31, 23-33.

61. Birchler, J.A. Genetic Rules of Heterosis in Plants. Polyploid and Hybrid Genomics, 2013. 313.

62. Dapp, M.; Reinders, J.; Bédiée, A.; Balsera, C.; Bucher, E.; Theiler, G.; Granier C.; Paszkowski, Jerzy. Heterosis and inbreeding depression of epigenetic Arabidopsis hybrids. Nat. Plants 2015. 1, 15092.

63. Guerin, J.; Rossel, J. B.; Robert, S.; Tsuchiya, T.; Koltunow, A. A. deficiens homologue is down-regulated during apomictic initiation in ovules of Hieracium. Planta 2000, 76, 914-920

64. Siena, L. A.; Ortiz, J. P. A.; Leblanc, O.; Pessino, A. S. PnTgs1-like expression during reproductive development supports a role for RNA methyltransferases in the aposporous pathway. BMC Plant Biol. 2014. 14, 297

65. Ferreira, L. G.; de Alencar Dusi, D. M.; Irsigler, A. S. T.; Gomes, A.; Mendes, M. A.; Colombo, L.; de Campos Carneiro, V. T. GID1 expression is associated with ovule development of sexual and apomictic plants. Plant Cell Rep. 2018, 37, 293-306.

66. Nonomura, K. I. The MSP1 gene is necessary to restrict the number of cells entering into male and female sporogenesis and to initiate anther wall formation in rice. The Plant Cell Online 2003, 15, 1728-1739.

67. Podio, M.; Caceres, M. E.; Samoluk, S. S.; Seijo, J. G.; Pessino, S. C.; Ortiz, J. P.; Pupilli, F. A methylation status analysis of the apomixisspecific region in Paspalum spp. suggests an epigenetic control of parthenogenesis. J. Exp. Bot. 2014, 65, 6411-6424.

68. Siena, L. A.; Ortiz, J. P.; Calderini, O.; Paolocci, F.; Caceres, M. E.; Kaushal, P.; Grisan, S.; Pessino, S. C.; Pupilli, F. An apomixis-linked ORC3-like pseudogene is associated with 
silencing of its functional homolog in apomictic Paspalum simplex. J. Exp. Bot. 2016, 67, 1965-1978.

69. Liu, D. D.; Dong, Q. L.; Sun, C.; Wang, Q. L.; You, C. X.; Yao, Y. X.; Hao, Y. J. Functional characterization of an apple apomixisrelated MhFIE gene in reproduction development. Plant Sci. 2012, 185-186.

70. Boateng, K. A.; Yang, X.; Dong, F.; Owen, H. A.; Makaroff, C. A. SWII is required for meiotic chromosome remodeling events. Mol. Plant 2008, 1, 620-633.

71. Zhao, L.; He, J.; Cai, H.; Lin, H.; Li, Y.; Liu, R.; Yang, Z.; Qin, Y. Comparative expression profiling reveals gene functions in female meiosis and gametophyte development in Arabidopsis. Plant J. 2014, 80, 615-628.

72. Olmedo-Monfil, V.; Figueroa, N.D.; Vázquez, M.A.; Arévalo,M.D.; Autran, D.; Grimanelli, D.; Slotkin, R.K.; Martienssen, R.A.; Calzada,J.P.V. Control of female gamete formation by a small RNA pathway in Arabidopsis. Nature 2010. 464, 628.

73. Hehenberger, E.; D. Kradolfer, D.; Köhler, C. Endosperm cellularization defines an important developmental transition for embryo development. Development 2012. 139, 2031-2039.

74. Felitti, S.A.; Seijo, J.G.; González,A.M.; Podio, M.; Laspina, N.V.; Siena, L.; Ortiz, J.P.A.; Pessino, S.C.; Expression of lorelei-like genes in aposporous and sexual Paspalum notatum plants. Plant Mol. Biol. 2011. 77, 337.

75. Garcia-Aguilar, M.; Michaud, C.; Leblanc, O.; Grimanelli, D. Inactivation of a DNA methylation pathway in maize reproductive organs results in apomixis-like phenotypes. Plant Cell 2010. 22, 3249-3267. 
Table 1. Apomixis related candidate genes and related information.

\begin{tabular}{|c|c|c|c|c|}
\hline Genes & $\begin{array}{l}\text { Affected } \\
\text { process } \\
\end{array}$ & Description & Species & References \\
\hline$A S G R-B B M L$ & Apospory & $\begin{array}{l}\text { Multiple copies of the } \\
\text { PsASGRBABY BOOM-like } \\
(P S A S G R B B M L) \text { gene } \\
\text { reside within the apospory- } \\
\text { specific genomic region }\end{array}$ & $\begin{array}{l}\text { Pennisetum } \\
\text { squamulatum } \\
\text { (apospory) }\end{array}$ & [9] \\
\hline $\begin{array}{l}\text { LOSS OF APOMEIOSIS } \\
(L O A)\end{array}$ & Apospory & Responsible for apospory & $\begin{array}{l}\text { Hieracium Praealtum } \\
\text { (apospory) }\end{array}$ & {$[55]$} \\
\hline Deficiens & Apospory & $\begin{array}{l}\text { Deficiens is a member of } \\
\text { the B class of floral } \\
\text { homeotic regulators that } \\
\text { have been shown to } \\
\text { specify petal and stamen } \\
\text { identity according to the } \\
\text { ABC model of floral } \\
\text { development }\end{array}$ & $\begin{array}{l}\text { Hieracium } \\
\text { piloselloides } \\
\text { (apospory) }\end{array}$ & {$[63]$} \\
\hline PnTgsl-like & Apospory & $\begin{array}{l}\text { PnTgs1-like (formerly } \\
\text { N69) encodes a } \\
\text { trimethylguanosine } \\
\text { synthase-like protein } \\
\text { whose function in } \\
\text { mammals and yeast is } \\
\text { critical for development, } \\
\text { including reproduction }\end{array}$ & $\begin{array}{l}\text { Paspalum notatum } \\
\text { (obligate apomixis) }\end{array}$ & {$[64]$} \\
\hline GID1 & Apospory & $\begin{array}{l}\text { Gibberellin-insensitive } \\
\text { DWARF1 }\end{array}$ & $\begin{array}{l}\text { Brachiaria brizantha } \\
\text { (apospory) }\end{array}$ & {$[65]$} \\
\hline$M S P 1$ & $\begin{array}{l}\text { Adventitious } \\
\text { embryony }\end{array}$ & $\begin{array}{l}\text { Multiple sporocyte } \\
\text { controls early sporogenic } \\
\text { development }\end{array}$ & $\begin{array}{l}\text { Oryza sativa } \\
\text { (amphimixis) }\end{array}$ & {$[66]$} \\
\hline$R W P$ & $\begin{array}{l}\text { Adventitious } \\
\text { embryony }\end{array}$ & $\begin{array}{l}\text { RWP is the key gene } \\
\text { controlling polyembryony }\end{array}$ & $\begin{array}{l}\text { Citrus reticulate } \\
\text { (sporophyte apomixis) }\end{array}$ & {$[16]$} \\
\hline SERK & $\begin{array}{l}\text { Adventitious } \\
\text { embryony }\end{array}$ & $\begin{array}{l}\text { Somatic embryogenesis } \\
\text { receptor-like kinase }\end{array}$ & $\begin{array}{l}\text { Poa pratensis } \\
\text { (amphimixis or } \\
\text { apomixis) / Paspalum } \\
\text { notatum (obligate } \\
\text { apomixis) }\end{array}$ & {$[67]$} \\
\hline ORC & $\begin{array}{l}\text { Adventitious } \\
\text { embryony }\end{array}$ & $\begin{array}{l}\text { ORC (ORIGIN } \\
\text { RECOGNITION } \\
\text { COMPLEX) is a } \\
\text { multiprotein complex } \\
\text { which controls DNA } \\
\text { replication and cell } \\
\text { differentiation in } \\
\text { eukaryotes }\end{array}$ & $\begin{array}{l}\text { Paspalum simplex } \\
\text { (apospory) }\end{array}$ & {$[68]$} \\
\hline FIE & $\begin{array}{l}\text { Autonomous } \\
\text { endosperm }\end{array}$ & $\begin{array}{l}\text { Fertilization independent } \\
\text { endosperm }\end{array}$ & $\begin{array}{l}\text { Malus hupehensis } \\
\text { (facultative apomictic } \\
\text { reproduction) } \\
\text { /Solanum } \\
\text { lycopersicum } \\
\text { (amphimixis) }\end{array}$ & [69] \\
\hline
\end{tabular}




\begin{tabular}{|c|c|c|c|}
\hline DYAD/SWITCH1 & Diplospory & $\begin{array}{l}\text { Result in defects in female } \\
\text { meiosis/ affect meiosis in } \\
\text { megasporocytes }\end{array}$ & $\begin{array}{l}\text { Arabidopsis, dyad } \\
\text { mutant (amphimixis) }\end{array}$ \\
\hline Apostart & Diplospory & $\begin{array}{l}\text { Speculated to participate in } \\
\text { the formation of } 2 \mathrm{n} \text { eggs in } \\
\text { apomixis }\end{array}$ & $\begin{array}{l}\text { Medicago sativa } \\
\text { (diplospory) }\end{array}$ \\
\hline$D M C 1$ & Diplospory & Participation in meiosis & $\begin{array}{l}\text { Arabidopsis } \\
\text { (amphimixis) }\end{array}$ \\
\hline Argonaute 9 (AGO 9) & Diplospory & $\begin{array}{l}\text { AGO9 controls female } \\
\text { gamete formation by } \\
\text { limiting the specification } \\
\text { of gametophyte precursors } \\
\text { in a non-cell autonomous } \\
\text { manner }\end{array}$ & $\begin{array}{l}\text { Arabidopsis, AGO9 } \\
\text { mutant (diplospory) }\end{array}$ \\
\hline AGO 104 & Diplospory & $\begin{array}{l}\text { AGO } 104 \text { and } A G O 9 \text { are } \\
\text { homologous genes, and } \\
\text { their defects can produce a } \\
\text { phenotype similar to } \\
\text { apomixis, giving rise to up } \\
\text { to } 70 \% \text { of functional } \\
\text { unreduced female gametes }\end{array}$ & $\begin{array}{l}\text { Tripsacum } \\
\text { (diplospory) }\end{array}$ \\
\hline AGAMOUS-LIKE 62 & $\begin{array}{l}\text { Inhibit endosperm } \\
\text { cellularization }\end{array}$ & $\begin{array}{l}\text { Agamous-like } 62 \text { (AGL62) } \\
\text { is a member of the MADS- } \\
\text { box gene, an increase in its } \\
\text { expression level can } \\
\text { inhibit the development of } \\
\text { the germ and inhibit } \\
\text { endosperm cellularization }\end{array}$ & $\begin{array}{l}\text { Arabidopsis, fis } 2 \\
\text { mutant (amphimixis) }\end{array}$ \\
\hline lorelei-like/n20gap-1 & $\begin{array}{l}\text { Might play a role in } \\
\text { the final stages of } \\
\text { the apomixis } \\
\text { developmental } \\
\text { cascade. }\end{array}$ & $\begin{array}{l}\text { Encodes a GPI-anchored } \\
\text { protein previously } \\
\text { associated with apomixis. }\end{array}$ & $\begin{array}{l}\text { Paspalum notatum } \\
\text { (obligate apomixis) }\end{array}$ \\
\hline$d m t$ & $\begin{array}{l}\text { May be involved in } \\
\text { differentiation } \\
\text { between apomictic } \\
\text { and sexual } \\
\text { reproduction }\end{array}$ & $\begin{array}{l}\text { The expression of the dmt } \\
\text { (DNA methyl transferases) } \\
\text { gene was significantly } \\
\text { different between sexual } \\
\text { and apomictic } \\
\text { reproduction. The absence } \\
\text { of dmt102 and dmt } 103 \text { can } \\
\text { produce a phenotype } \\
\text { similar to apomixis }\end{array}$ & $\begin{array}{l}\text { maize-Tripsacum } \\
\text { hybrid (diplospory) }\end{array}$ \\
\hline
\end{tabular}

[70] 

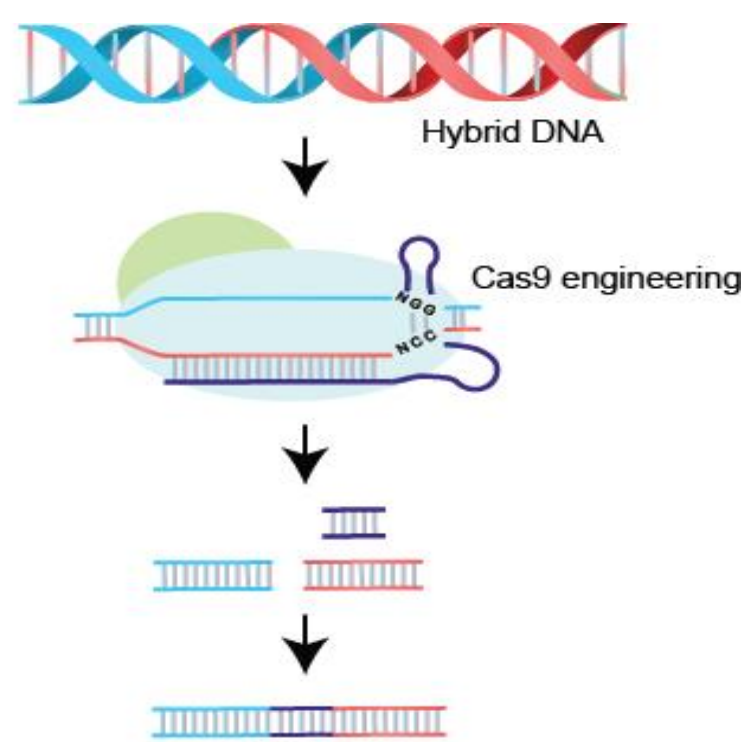

Clonal hybrid DNA
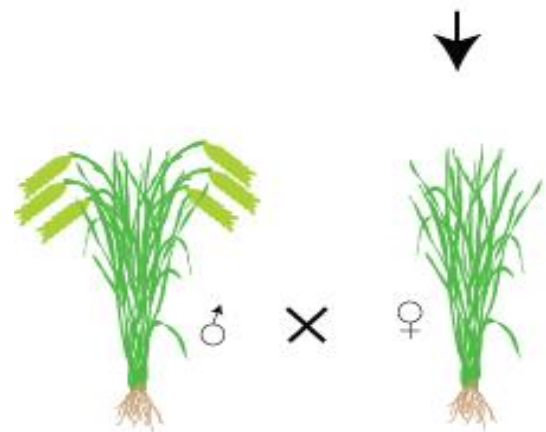

Restorer

Sterile (P/TGMS) plant

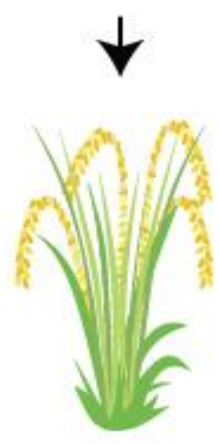

Fi hybrid

Figure 1. Schematic description of the two line hybrid development utilizing CRISPR/Cas system. P/TGMS = photoperiod/thermo-sensitive genic male sterile. 


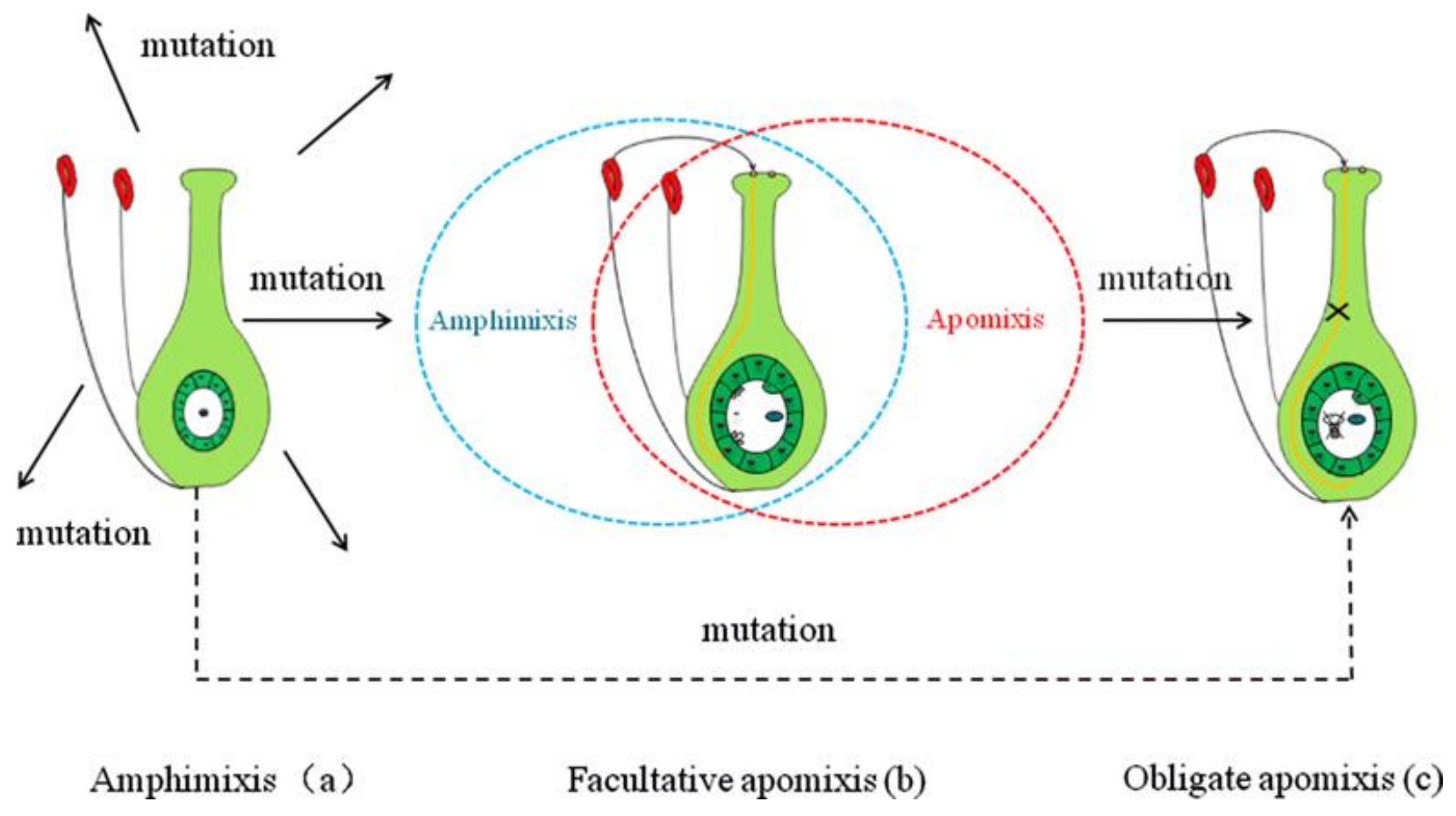

Figure 3. The emergence of apomictic types. (a) Amphimixis (b) Facultative apomixis and (c) Obligate apomixis. (Fie et al., [30]). 

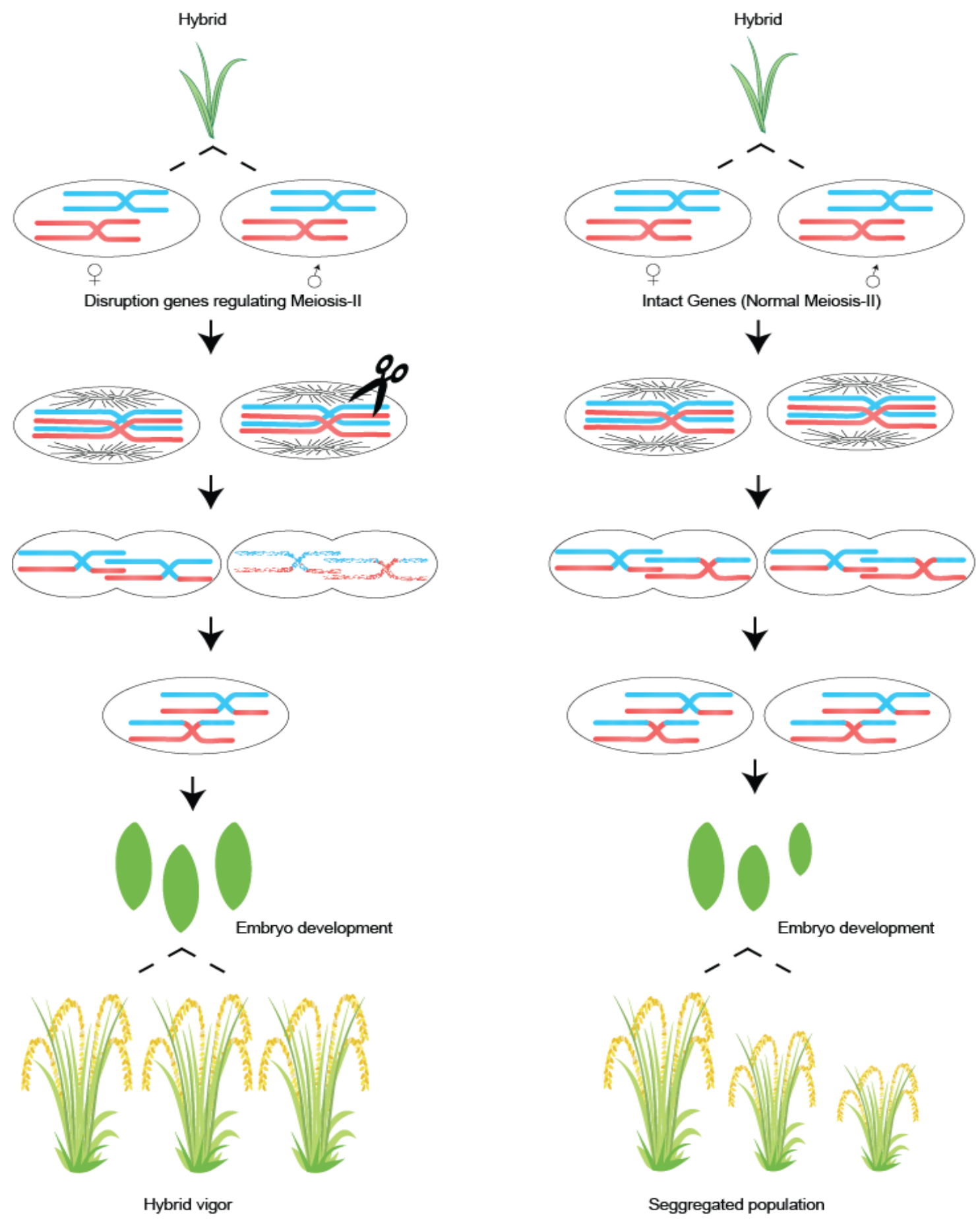

Figure 4. An illustration of promoting apomixis for hybrid vigour preservation through the CRISPR/Cas9 system. Hybrid development took place via genome editing of genes or through conventional breeding techniques. To fix hybrid vigor in $\mathrm{F}_{2}$ scientist are promoting apomixis in hybrid seed to keep intact the characteristics of $\mathrm{F}_{1}$ hybrid seed. The MiMe phenotypes is achieved through disruption of gene controlling meiosis II which leads towards unreduced embryo sac essential for apomeiosis. However, in other case the hybrid segregate in $\mathrm{F}_{2}$ plant populations and all the important characteristics are lost. 


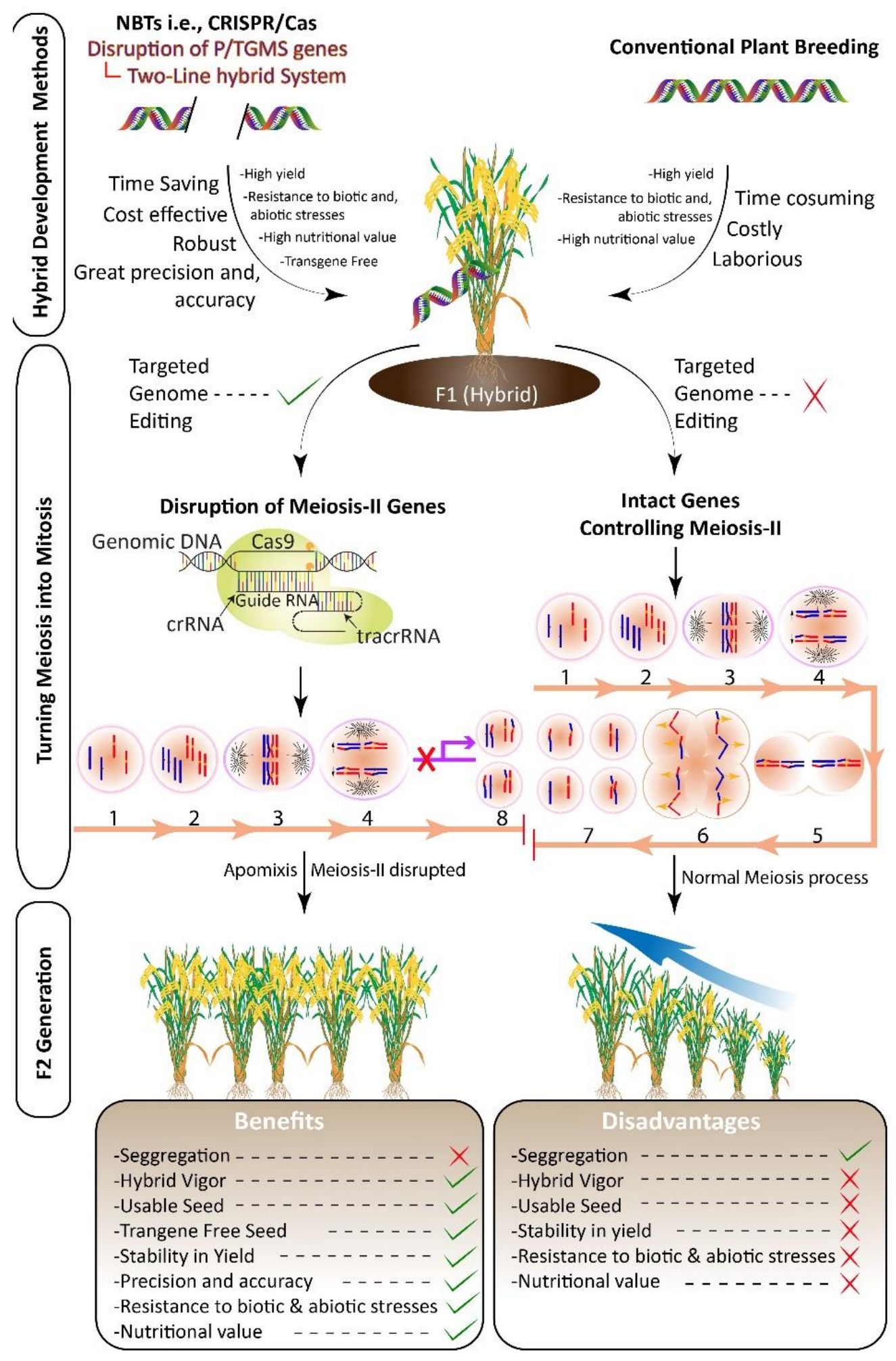

Figure 5. Summative illustration of promoting induced apomixis for hybrid vigour preservation through the CRISPR/Cas9 genome editing system. Hybrid development took place via genome editing of genes or through conventional breeding techniques. To fix hybrid vigor in F2 scientist 
are promoting apomixis in hybrid seed to keep intact the characteristics of F1 hybrid seed. The MiMe phenotypes is achieved through disruption of gene controlling meiosis II which leads towards unreduced embryo sac essential for apomeiosis. However, in other case the hybrid segregate in F2 plant populations and all the important characteristics are lost. 1, Interphase; 2, Prophase-I; 3, Metaphase-I; 4, Anaphase-I; 5, Metaphase-II; 6, Anaphase-II; 7, n gametes; 8, 2n gametes; NBTs, new breeding techniques; P/TGMS, photoperiod/thermo-genic male sterile; Cas9; CRISPR- associated protein 9, tracrRNA (trans-activating RNA). 